\title{
Management of Mediastinal Pancreatic Fluid Collections by Endoscopic Pancreatic Ductal Decompression
}

\author{
Weijie Yao ${ }^{1}$, Shiyang $\mathrm{Ma}^{2}$, Wenping $\mathrm{Bo}^{1}$ and Zuozheng Wang ${ }^{1 *}$ \\ ${ }^{1}$ Department of Hepatobiliary Surgery of the General Hospital of NingXia Medical University, China \\ ${ }^{2}$ Department of Gastroenterology, the Second Affiliated Hospital of Xi'an Jiaotong University, China
}

*Corresponding author: Zuozheng Wang, Department of Hepatobiliary Surgery, General Hospital of Ningxia Medical University, China

\begin{abstract}
ARTICLE INFO
Received: 蔧 June 11, 2020

Published: 幽 June 22, 2020

Citation: Weijie Yao, Shiyang Ma, Wenping Bo, Zuozheng Wang. Management of Mediastinal Pancreatic Fluid Collections by

ABSTRACT

The mediastinum is an uncommon site for peri-pancreatic fluid collections. Between September 2017 and May 2018, a total of 3 patients developed mediastinal pancreatic fluid collections after pancreatitis in our center. After endoscopic main pancreatic duct decompression, all patient recovered uneventfully with the disappear of mediastinal pancreatic fluid collection. We suggest endoscopic main pancreatic duct decompression is a safe and efficient treatment in the management of mediastinal collections associated with pancreatic diseases.
\end{abstract} Endoscopic Pancreatic Ductal Decompression. Biomed J Sci \& Tech Res 28(3)-2020. BJSTR. MS.ID.004650.
Keywords: Peri-Pancreatic Fluid Collections; Endoscopy; Mediastinal; Pancreatic Stent

\section{Introduction}

Acute peri-pancreatic fluid collection and pancreatic pseudocyst can develop after pancreatitis. However, the mediastinum is an uncommon site for peri-pancreatic fluid collections. In mediastinal pancreatic fluid collection, drainage of pancreatic juice or the abdominal component of the fluid collection should be considered

\section{Case Series}

Table 1: The patients' demographics, presentation, clinical finding and management.

\begin{tabular}{|c|c|c|c|}
\hline & Case 1 & Case 2 & Case 3 \\
\hline Age/Sex & $61 /$ man & $75 /$ man & Greasy food \\
\hline Etiology & Alcohol abuse & Unknown & Chronic \\
\hline Acute/Chronic pancreatitis & Chronic & Acute & No \\
\hline Abdominal pancreatic pseudocyst & Yes & Pleural effusion & Pleural effusion \\
\hline Associated complications & Pleural effusion & & 8.1 \\
\hline Pulmonary infection & & 5.64 & 121 \\
\hline White cell count $(\times 109 / \mathrm{L})$ & 6.27 & 151 & 232 \\
\hline HGB $(\mathrm{g} / \mathrm{L})$ & 120 & 126 & \\
\hline PLT $(\times 109 / \mathrm{L})$ & 250 & & \\
\hline
\end{tabular}




\begin{tabular}{|c|c|c|c|}
\hline PT (s) & 13.6 & 13.5 & 15.9 \\
\hline ALT (U/L) & 45.6 & 30 & 7.7 \\
\hline AST (U/L) & 23 & 25.8 & 9.8 \\
\hline ALB (g/L) & 28 & 34.8 & 17.25 \\
\hline TBIL $(\mu \mathrm{mol} / \mathrm{L})$ & 15.6 & 12.4 & 6.77 \\
\hline DBIL $(\mu \mathrm{mol} / \mathrm{L})$ & 5.8 & 5.8 & 1.24 \\
\hline TG $(\mathrm{mmol} / \mathrm{L})$ & 0.73 & 5.25 & 5.57 \\
\hline GLU $(\mathrm{mmol} / \mathrm{L})$ & 7.5 & 2.24 & 1.87 \\
\hline Ca $(\mathrm{mmol} / \mathrm{L})$ & 2.07 & 384 & 1013 \\
\hline AMY (U/L) & 521 & 1394 & 4095 \\
\hline Lipase (U/L) & 1001 & 18 & 1.66 \\
\hline Ca199 (U/ml) & 5.8 & Pancreatic stenting & \\
\hline Endoscopic management & Naso-pancreatic tube & & \\
\hline pancreatic stenting & & & \\
\hline
\end{tabular}

Between September 2017 and May 2018, a total of 3 patients developed mediastinal pancreatic fluid collections after pancreatitis. The associated symptoms included severe epigastric pain that radiating to the back and mild chest tightness (the patients information shown in Table 1). The CT scans showed mediastinal fluid collections (Figure 1). Multiple pleural fluid test results showed significant increase in amylase. Despite standard supportive care that included total parenteral nutrition and treatment with proton pump inhibitor, octreotide, and ceftriaxone, the symptomatic fluid collections persisted. Subsequently, endoscopic main pancreatic duct decompression was performed by placement of nasobiliary tube or nasopancreatic tube, or pancreatic stent. All patient recovered uneventfully with the disappear of symptoms as well as mediastinal pancreatic fluid collection (Figure 1).

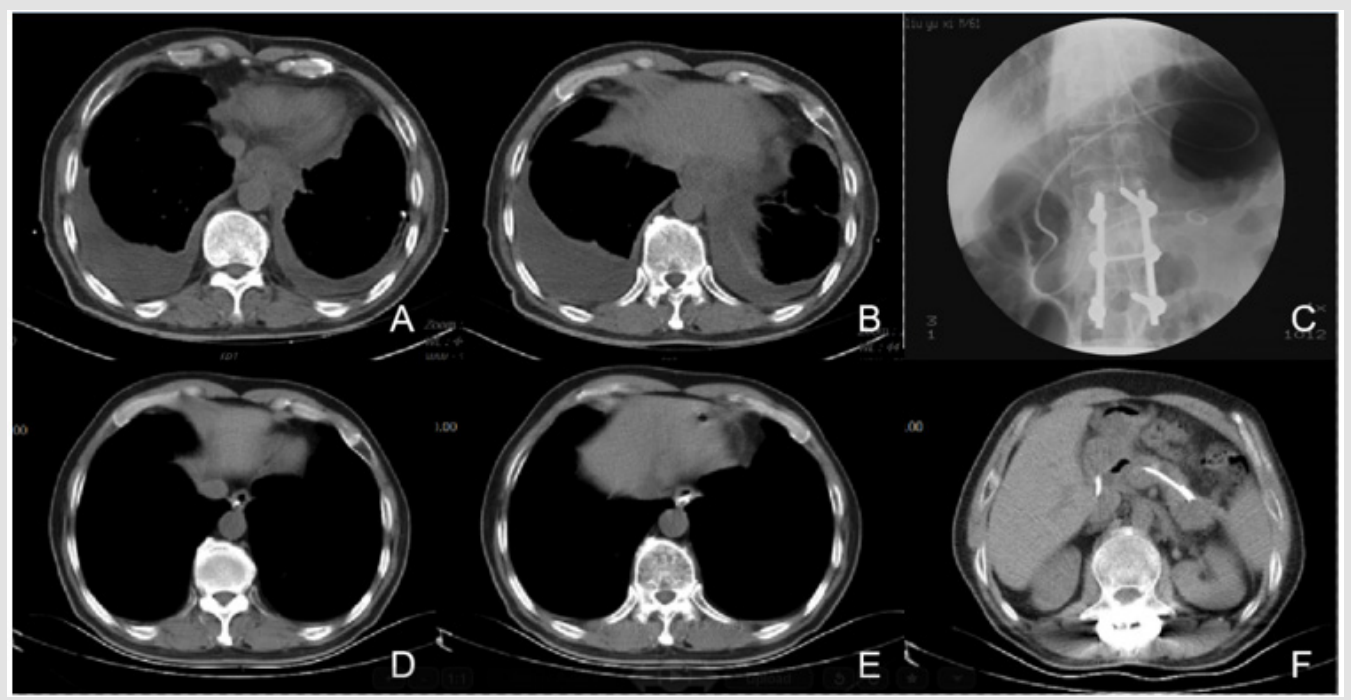

Figure 1: CT images

(A and B): Showed mediastinal fluid collections with external compression on the esophagus, bilateral pleural effusion.

(C): A nasobiliary tube is inserted into pancreatic pseudocyst through main pancreatic duct for drainage; (D and E): One month later, the fluid collection has resolved after the drainage;

(F): The position of drainage tube.

\section{Discussion}

Mediastinal pancreatic fluid collection is a rare complication resulting from chronic or acute pancreatitis and maybe the initial stage of mediastinal pseudocysts. Both of them belong to the category of thoracopancreatic fistula and occur as a result of posterior rupture of the pancreatic duct into the retroperitoneal space. In most cases, acute extra-pancreatic fluid collection infiltrates an already existing anatomic space, such as the lesser sac, the anterior para-renal space, the posterior para-renal space, around the left lobe of the liver, and in the spleen. Rarely, pancreatic fluid collections extend upward through anatomical openings of the diaphragm into the mediastinum. The fluids typically travel through the esophageal and aortic hiatus into the posterior mediastinum [1-2]. The diaphragm also could be penetrated through the inferior 
vena cava hiatus or the foramen of Morgagni, and pancreatic fluid extends into the middle and anterior mediastinum, respectively [34]. Mediastinal fluid collections can cause dysphagia, chest pain, dyspnea secondary to an accompanying pleural effusion. A history of pancreatitis or pancreatic pseudocyst may help in the diagnostic workup. Sometimes, it was difficult to suspect pancreatitis in all mediastinal fluid collections. CT scan is most commonly used to establish the diagnosis, and it also helps to depict pancreatitis and pancreatic pseudocyst [5]. The connection between any mediastinal cystic structures and the pancreas may be identified by sagittal reconstruction [6]. MRI may provide further details of biliary and pancreatic ductal anatomy. Endoscopic Ultrasound (EUS) is helpful in evaluating these fluid collections and further play an important therapeutic role by EUS-guided aspiration and drainage of these fluid collections.

In our cases, chest and abdominal CT scans showed mediastinal fluid collections accompanied by pancreatic pseudocyst, pancreatic atrophy or focal pancreatitis, dilatation, and/or stricture of the main pancreatic duct. Bilateral pleural effusions were also seen in all cases. These are key findings of complicated acute pancreatitis. There might be connections at the esophageal hiatus, aortic hiatus or inferior vena cava hiatus. However, the connections between mediastinal and abdominal lesions through the diaphragm were not well identified as mediastinal pseudocysts. Furthermore, MRI and EUS may provide further details.

Management of mediastinal fluid collections depends on their size, quantity, the presence of infection or hemorrhage, and symptom severity. Spontaneous regression is rare but may happen after medical therapy, such as octreotide [7]. Draining pancreatic fluid and trans-papillary stenting of the pancreatic duct are useful treatments that reduce the high pressures associated with pseudocysts and pancreatic duct narrowing [8]. ERCP with transpapillary stenting of the pancreatic duct can be very effective while CT-guided or EUS-guided drainage may be other options. Surgical options include internal drainage, external drainage, cystenterostomy, or pancreatic resection. But surgery is invasive but can be considered when all other treatment modalities fail [9]. In our cases, the decompression of the main pancreatic duct successfully lead to mediastinal fluid collection resolution, which strongly support ERCP should be considered as the first-line treatment.

In conclusion, we present three cases of mediastinal fluid collections. Timely drainage and decompression of the main pancreatic duct are essential in managing these patients.

\section{Acknowledgement}

We gratefully acknowledge Dr. Shou-Jiang Tang for reading the manuscript.

\section{Conflicts of interest}

No conflicts of interest.

\section{Funding}

None.

\section{Statement of Ethics}

All patients were informed and agreed to write articles using their data.

\section{Author Contributions}

Weijie Yao and Wenping Bo are mainly involved in patient management; Shiyang Ma participated in the writing of the article; Zuozheng Wang performed all the endoscopic procedures.

\section{Conclusion}

In conclusion, placement of straight and single pig tail pancreatic stent needs maximal caution. In case of proximal stent migration, forceps removal can be attempted, especially when the migrated stent is embedded, and the PD is obstructed by the embedded stent.

\section{Acknowledgement}

We gratefully acknowledge Dr. Shou-Jiang Tang for reading the manuscript.

\section{References}

1. Leger L, Pagniez G, Lenriot JP (1970) Dysphagia revealing chronic pancreatitis. 2 cases of mediastinal pseudocysts. J Chir (Paris) 99(3): 217-234.

2. Valverde A, Hammel P, Zins M, Le Mée J, Bernades P, et al. (1999) Mediastinal and cervical diffusion of necrosis infiltration in acute pancreatitis. Gastroenterol Clin Biol 23(1): 137-140.

3. Trudzinski FC, Rädle J, Treiber G, Kramm T, Sybrecht GW (2008) Massive pleural effusion complicating chronic pancreatitis. Treatment by endoscopic closure of a pancreatic-mediastinal fistula. Dtsch Med Wochenschr 133(48): 2507-2509.

4. Xu H, Zhang X, Christe A, Ebner L, Zhang S, et al. (2013) Anatomic pathways of peripancreatic fluid draining to mediastinum in recurrent acute pancreatitis: Visible Human Project and CT Study. PLoS One 8(4): e62025.

5. Di Cesare E, Di Sibio A, Gennarelli A, Felli V, Vellucci V, et al. (2014) An Unusual Case of Ascending Pancreatitis with Mediastinal Involvement: A Case Report with CT and MRI Findings. Case Rep Radiol 2014: 925105.

6. Visrutaratna P, Ukarapol N (2010) Mediastinal pancreatic pseudocyst in chronic pancreatitis. Pediatr Radiol 40: 1298.

7. Santoshkumar S, Seith A, Rastogi R, Khilnani GC (2007) Mediastinal pseudocysts in chronic pancreatitis with spontaneous resolution. Trop Gastroenterol 28(1): 32-34

8. Mishra SK, Jain PK, Gupta S (2016) Mediastinal Pseudocyst in Acute on Chronic Pancreatitis. J Assoc Physicians India 64: 80-81.

9. Rana SS, Sharma V, Reddy S, Bhasin DK (2015) Combined endovascular and endoscopic management of thoracic aortic pseudoaneurysm, mediastinal pseudocyst, and pancreatic pleural effusion due to chronic pancreatitis. Gastrointest Endosc 81(6): 1501-1502. 
ISSN: 2574-1241

DOI: $10.26717 / B J S T R .2020 .28 .004650$

Zuozheng Wang. Biomed J Sci \& Tech Res

(C) (i) This work is licensed under Creative Submission Link: https://biomedres.us/submit-manuscript.php

\begin{tabular}{ll}
\hline BIOMEDICAL & Assets of Publishing with us \\
RESERCHES & - Global archiving of articles \\
- Immediate, unrestricted online access & - Rigorous Peer Review Process \\
\hline https://biomedres.us/
\end{tabular}

